\title{
Depressive Symptoms during Pregnancy and the Concentration of Fatty Acids in Breast Milk
}

\author{
Sarah A. Keim, PhD, MA, MS ${ }^{1,2}$, Julie L. Daniels, PhD, MPH ${ }^{1,3}$, Anna Maria Siega-Riz, PhD, \\ MS $^{1,4,5}$, Nancy Dole, PhD ${ }^{1,5}$, Amy H. Herring, ScD ${ }^{5,6}$, and Peter C. Scheidt, MD, MPH ${ }^{2,7}$ \\ ${ }^{1}$ Department of Epidemiology, University of North Carolina at Chapel Hill, Chapel Hill, North \\ Carolina ${ }^{2}$ National Children's Study, Eunice Kennedy Shriver National Institute of Child Health \\ and Human Development, Bethesda, Maryland ${ }^{3}$ Department of Maternal and Child Health, \\ University of North Carolina at Chapel Hill, Chapel Hill, North Carolina ${ }^{4}$ Department of Nutrition, \\ University of North Carolina at Chapel Hill, Chapel Hill, North Carolina ${ }^{5}$ Carolina Population \\ Center, University of North Carolina at Chapel Hill, Chapel Hill, North Carolina ${ }^{6}$ Department of \\ Biostatistics, University of North Carolina at Chapel Hill, Chapel Hill, North Carolina
}

\section{Abstract}

The aim of the present study was to examine the association between depressive symptoms in pregnancy and the concentration of long-chain polyunsaturated fatty acids (LCPUFAs) in breast milk. Women $(n=287)$ enrolled in the Pregnancy, Infection, and Nutrition Study completed the Center for Epidemiologic Studies Depression Scale in pregnancy ( $<20$ and 24-29 weeks) and had LCPUFAs measured in breast milk (4 months postpartum). Multiple linear regression was used to examine associations between depressive symptoms and breast milk LCPUFAs. Increasing depressive symptoms at $<20$ weeks were associated with lower docosahexaenoic acid concentrations (adjusted $\beta=-1.15,95 \%$ confidence interval $=-2.12,-0.19$ ). No similar associations were observed with other fatty acids nor between symptoms at 24-29 weeks and LCPUFAs. Depressive symptoms, even in the subclinical range, early in pregnancy are inversely associated with breast milk docosahexaenoic acid. This may have implications for the timing of screening and interventions for perinatal depression and the nutritional value of breast milk.

\section{Keywords}

depression; postpartum depression; fatty acids; pregnancy; breast milk; introduction

\begin{abstract}
Pregnancy and the postpartum period can be stressful for women; depression affects an estimated $8 \%$ to $11 \%$ of women at some point during pregnancy. ${ }^{1}$ Long-chain polyunsaturated fatty acids (LCPUFAs), particularly docosahexaenoic acid (DHA), appear to play a role in maintaining positive mental health, although the biological mechanism remains unclear. ${ }^{2}$ Low levels of DHA have been noted in the red blood cell and plasma cell membranes and brain orbitofrontal cortex tissue of clinically depressed adults. ${ }^{3-5}$

Additionally, DHA is important for optimal brain development early in life. ${ }^{6}$ Pregnancy and lactation may exacerbate low DHA status because maternal DHA stores are mobilized to
\end{abstract}

(C) The Author(s) 2012

Corresponding Author: Sarah A. Keim, 700 Children's Dr, Columbus, OH 43205. keim.22@osu.edu.

${ }^{7}$ Children's National Medical Center, Washington, DC (current affiliation)

Declaration of Conflicting Interests

The authors declared no potential conflicts of interest with respect to the research, authorship, and/or publication of this article. 
support the rapid development of the fetal and infant brain. ${ }^{7}$ Some clinical trials have evaluated whether $\omega-3$ fatty acid supplements prevent perinatal or postpartum depression or reduce symptoms, with mostly negative findings. ${ }^{8-12}$ A handful of observational studies have examined plasma DHA levels among women with perinatal or postpartum depression, but the results have been inconsistent, perhaps due to limited sample sizes. ${ }^{13-16}$ Most of these studies dichotomized scores on depressive symptoms assessments or selected women who met a clinical definition for depression, so they were unable to examine associations at subclinical levels. In addition, previous studies have focused on only the third trimester or postpartum period, despite indications that many women with postpartum depression experience symptoms early in pregnancy. ${ }^{17}$

No previous studies have examined the relationship between depressive symptoms during pregnancy and breast milk fatty acid concentrations, regardless of how common elevated depressive symptoms are during pregnancy. Even though breast milk and plasma fatty acid concentrations are generally positively correlated, examination of breast milk DHA levels is important because breast milk is more reflective of fatty acid stores laid down over a lifetime and of lifetime diet than short-term influences (one study estimated 29\% of breast milk fatty acids derive from current diet and 59\% derive from adipose tissue) and because of breast milk's role as a LCPUFA source for breastfed infants. ${ }^{18-22}$ Most previous observational studies measured LCPUFAs in plasma samples collected at the same time as screening or diagnosis of depression. The inability to establish the temporality of the association makes it unclear whether lower DHA is a cause, a noncausal correlate, or a result of depression. That is, low DHA levels, either because of low dietary intake or abnormal metabolism of precursor fatty acids, could affect cell membrane phospholipid composition and function in the brain and cause depression. Conversely, depression could cause reduced dietary intake of healthy $\omega$-3-rich foods or affect fatty acid metabolism to drive DHA levels lower. Previous cross-sectional designs could not explore these issues. Additionally, low DHA status and depression could be related through one or more confounding factors, such as socioeconomic status or genetic factors, that would explain an observed association. Because depression is often a chronic condition and fatty acid status is at least partly reflective of lifetime diet, even longitudinal studies are challenged to sort out the temporality of these phenomena, but they can verify the presence of one factor (eg, depressive symptoms) at a point in time at least as early as a second factor (eg, fatty acid levels). We addressed this objective by analyzing data from the longitudinal Pregnancy, Infection, and Nutrition (PIN) study to examine the relationship between depressive symptoms at 2 times during pregnancy, as measured with the Center for Epidemiologic Studies Depression Scale (CES-D) and the LCPUFA content of breast milk collected at 4 months postpartum.

\section{Methods}

\section{Study Population}

Data were from the PIN study and its postnatal follow-up component, PIN Postpartum. The design and goals of these studies have been described. ${ }^{23,24}$ All protocols were approved by the University of North Carolina at Chapel Hill Biomedical Institutional Review Board; all participants provided written informed consent.

Women ( $\mathrm{n}=1169$ ) were eligible for PIN Postpartum if they completed the PIN study (phase 3 ), agreed to be contacted after delivery, and lived in the area. Exclusions for medical constraints $(n=24)$, inability to recontact $(n=207)$ or schedule $(n=62)$, and refusal $(n=187)$ resulted in 689 women who completed a visit in the fourth postpartum month. Women were eligible to provide a breast milk sample if they gave birth January 2004 to December 2006 $(n=519)$ and were breastfeeding and living in the area (165 women never initiated or had discontinued breastfeeding before the visit). Some women were unable to provide a sample 
$(\mathrm{n}=26)$ or declined $(\mathrm{n}=23)$. Of the 305 who provided a sample, 8 did not participate in either depressive symptoms assessment, 9 had 1 to 2 fatty acid values that could not be calculated, and 1 had an unanalyzable sample, leaving 287 women for inclusion in this analysis.

\section{Data Collection}

During pregnancy, information about depressive symptoms was collected via selfadministered questionnaires (CES-D scale) before 20 weeks and at 24-29 weeks of pregnancy. ${ }^{25}$ Women were asked to use a breast pump around 10 ам on the day of the postpartum visit, empty both breasts, mix the milk, and aliquot into $31.5-\mathrm{mL}$ tubes of milk for storage at $-80^{\circ} \mathrm{C}, 1$ of which was sampled for this study. Samples collected before April 1, 2005, were analyzed by the Collaborative Studies Clinical Laboratory at the University of Minnesota Medical Center, Fairview (Minneapolis, Minnesota). Those collected after April 1, 2005, were analyzed by the Clinical Nutrition Research Center, University of North Carolina (Chapel Hill, North Carolina). Fatty acid extraction was performed on $0.5-\mathrm{mL}$ samples of milk mixed with $0.5 \mathrm{~mL}$ of $0.9 \%$ saline using the method of Bligh and Dyer. ${ }^{26}$ Individual fatty acids were identified by comparison with authentic standards (Nu Chek Prep, Elysian, Minnesota). Data were analyzed with chromatography software (TotalChrom 6.2, PerkinElmer, Somerset, New Jersey).

Age, education, smoking, household income, and other demographic and lifestyle covariates were obtained by telephone at 17 to 30 weeks of pregnancy. At 24 to 29 weeks of pregnancy, women completed a modified Block Food Frequency Questionnaire, from which estimated daily dietary intake of $\omega-3$ and $\omega-6$ fatty acids was calculated for the 3 previous months. ${ }^{27}$

\section{Statistical Analysis}

We examined bivariate associations between continuous depressive symptoms scores measured at each time point and covariates and breast milk fatty acid concentrations (DHA, eicosapentaenoic acid [EPA], total $\omega-3$, total $\omega-6, \omega-6: \omega-3$ ratio) with Pearson correlation coefficient and $t$ tests. CES-D scores in continuous form were used to examine associations across the range of symptoms. Fatty acid variables (except $\omega-6: \omega 3$ ) were approximately lognormally distributed, so they were log-transformed for use in multiple linear regression models. We included a quadratic term for CES-D score in the models to examine whether it would improve model fit. To evaluate potential bias, we used $t$ tests to examine the difference among the CES-D scores for women who quit or never initiated breastfeeding or did not provide a breast milk sample.

A variable indicating which laboratory analyzed the sample was included in all regression models to adjust for inter-laboratory variability. Potential covariates were identified a priori on the basis of previous studies. Multiple partial $F$ tests assessed the presence of effect modification. Confounders in adjusted models included education, smoking during pregnancy, maternal age, parity, income as a percentage of the federal poverty level, and estimated daily dietary intake of $\omega-3$ or $\omega-6$ fatty acids (depending on the model). Results of regression models were backtransformed for interpretation (ie, percentage change in geometric mean fatty acid concentration).

\section{Results}

Participant characteristics are displayed in Table 1 . Nearly $12 \%$ of women scored $16+$ on the CES-D (a traditional cut point for high depressive symptoms) at less than 20 weeks of pregnancy; $16 \%$ scored $16+$ at 24 to 29 weeks of pregnancy. Of those who completed both 
assessments, $7 \%$ scored $16+$ both times. More depressive symptoms (results shown for symptoms at $<20$ weeks of pregnancy) were associated with fewer years of education ( $r=$ $-0.25, P<.01)$, smoking $(t=-6.5, P<.01)$, lower income $(r=-0.12, P=.04)$, and younger maternal age $(r=-0.20, P<.01)$. None of the covariates was a notable effect modifier. The food frequency questionnaire we used limited our ability to estimate daily dietary intake of individual $\omega-3$ fatty acids. However, compared to the only existing Institute of Medicine recommendation for $\omega-3$ fatty acids - which is for $\mathbf{a}$-linolenic acid alone (adequate intake $=$ $1.4 \mathrm{~g} /$ day for pregnant women) - the median intake of total $\omega-3$ fatty acids in this cohort was low (median $=0.42 \mathrm{~g} /$ day) $($ Table 2$) .{ }^{28}$ Finally, $\omega-3$ fatty acid intake was not associated with depressive symptoms.

All breast milk samples had fatty acid values above the limit of detection. Women with more education had higher breast milk DHA and EPA concentrations than women with less education: median DHA for women with 0 to 12 years $=0.14 \%(\mathrm{IQR}=0.09),>16$ years $=$ $0.25 \%(\mathrm{IQR}=0.25), P<.01$; median EPA for 0 to 12 years $=0.05 \%(\mathrm{IQR}=0.02),>16$ years $=0.09 \%(\mathrm{IQR}=0.09), P<.01$. Median DHA and EPA concentrations also differed by laboratory $(0.20 \%$ vs $0.39 \%$ DHA, $P<.01)$, although the distributions overlapped and the Spearman rank correlation coefficient based on 5 samples analyzed by both laboratories was 0.7 for DHA. Fatty acid concentrations in milk did not vary by estimated prenatal $\omega-3$ and $\omega-6$ intake from the food frequency questionnaire, age, income, or smoking (all $P>.10$ ). Women who quit or never initiated breastfeeding or did not provide a breast milk sample scored slightly higher on the CES-D at both time points (mean CES-D score at $<20$ weeks of pregnancy for those without a sample $=12.7 \pm 10.0$; with a sample $=9.2 \pm 7.1, t=-4.9, P$ $<.01$.

Depressive symptoms at $<20$ weeks of pregnancy, but not at 24 to 29 weeks, were inversely associated with DHA and total $\omega-3$ (Figure 1, Table 3). The association between depressive symptoms at 20 weeks and DHA remained after controlling for age, education, income, smoking, parity, $\omega-3$ intake, and laboratory: a 5-point increase in CES-D score corresponded to a $5.8 \%$ decrease in DHA. The association between depressive symptoms and total $\omega-3$ was attenuated upon adjustment. The quadratic term for CES-D was not significant in any model. Depressive symptoms were unassociated with EPA, total $\omega-6$, and $\omega-6: \omega-3$.

\section{Discussion}

The goal of this study was to examine whether depressive symptoms during pregnancy are inversely associated with breast milk LCPUFA concentrations. We found this to be true for DHA when depressive symptoms were measured at $<20$ weeks of pregnancy but not for symptoms measured at 24 to 29 weeks. The association with symptoms at $<20$ weeks applied across the range of CES-D scores; it appeared to be linear-that is, not confined to women scoring $16+$. We observed no associations between depressive symptoms at either time point and concentrations of other fatty acids in adjusted models.

Some proportion of women who experience elevated depressive symptoms late in pregnancy are women who have been chronically depressed and whose symptoms may be rooted in factors unrelated to and preceding the pregnancy, while other women have no history of depression but start to experience symptoms as hormonal triggers or psychosocial influences (eg, absence of a partner, financial worries) build as delivery nears. ${ }^{29}$ Our null results for symptoms measured around the beginning of the third trimester might be explained by the fact that women with elevated depressive symptoms detected early in pregnancy may be more reflective of the chronically depressed group, while the women with elevated symptoms later in pregnancy come from both these groups. Because breast milk DHA levels largely reflect fatty acid stores laid down over many years, low breast milk DHA may reflect 
chronic depression more closely than transient depressive symptoms during pregnancy. Again, it could be that (1) chronically low dietary intake or abnormal fatty acid metabolism could affect cell membrane phospholipid composition and function in the brain and cause depression or (2) chronic depression reinforces a diet low in $\omega-3$ fatty acids or adversely affects fatty acid metabolism as reflected in low $\omega-3$ levels. Alternatively, our positive result for depressive symptoms early in pregnancy could be spurious.

We evaluated the possibility that women who never initiated breastfeeding, who quit before 4 months, or who declined or were unable to provide a breast milk sample might have been more likely to have elevated depressive symptoms during pregnancy. These women scored slightly higher on the CES-D at both pregnancy time points. Because these women were not part of the analytic sample, our results may be biased toward the null and underestimate the true association.

No previous studies have examined depressive symptoms during pregnancy and breast milk fatty acid concentrations. One previous cross-sectional study of clinically depressed women found an inverse association between plasma DHA, total $\omega-3$, or $\omega-6: \omega-3$ and depressive symptoms in the third trimester, but it did not examine symptoms earlier in pregnancy, nor did it examine breast milk concentrations. ${ }^{16}$ Of several previous observational postpartum studies, 1 observed no association between postpartum depression and concurrent DHA status, while 2 observed that decreasing plasma DHA levels were accompanied by increasing depressive symptoms. ${ }^{13-15}$ Another study found an association on an ecologic level between national postpartum depression point prevalence estimates and breast milk composition reported by one or more studies aggregated in each of 16 countries. ${ }^{30}$ Our study is unique in that it examined the association between depressive symptoms and milk concentrations at the individual level.

By using a screening instrument to identify depressive symptoms, we included women with a range of depressive symptoms, rather than only women who met a clinical definition for major depression. Observing the association between DHA and depressive symptoms at subclinical levels suggests that a larger proportion of individuals might be affected, not only those who are clinically depressed. However, the use of this screener may underestimate the association among the most severely depressed women and is a limitation of our study. The proportion of women who experienced significant depressive symptoms in this study is similar to population-based studies of pregnancy, ${ }^{31,32}$ and the DHA concentration in the breast milk samples in this study was in the range of other US studies. ${ }^{33}$

Other limitations include the lack of information about depressive symptoms before pregnancy began. This information would have allowed us to examine depressive symptoms of a more chronic nature compared to newly emerging symptoms during pregnancy. Also, our limited sample size and relatively small number of women who experienced elevated symptoms at both pregnancy time points prevented in-depth analysis of the more severe symptoms levels.

While there is growing interest in the relation between depression and DHA, research has not revealed the temporal direction of the association. Most previous studies that examined the association between perinatal depressive symptoms and DHA status measured DHA at the same time or long before symptoms were measured. We examined the association longitudinally by assessing depressive symptoms during pregnancy and measuring fatty acid status postpartum. In this way, depressive symptoms present months before the measurement of DHA status may be meaningful, even if symptoms measured closer in time are not associated with DHA status. Because depressive symptoms often reflect a chronic condition and fatty acid levels are, at least in part, a product of lifetime diet, it is difficult for 
even a lengthy longitudinal study to resolve the temporal sequence of these factors.

However, this study found that depressive symptoms were present at a time point at least as early as the fatty acid profile (lower DHA). Additional research into the biological mechanisms underlying this association, as well as long prospective studies, will help clarify these uncertainties over temporality and causality.

\section{Conclusion}

The results of this study indicate that depressive symptoms early in pregnancy are associated with postpartum breast milk concentrations (symptoms may precede lower DHA status by months) and that even subclinical depressive symptoms may be associated with reduced concentrations of DHA in breast milk. However, additional large longitudinal studies are needed to trace the course of depressive symptoms and fatty acid status over longer periods. Perinatal depression is one of the most common mental health conditions during pregnancy and lactation, and it is a concern for clinicians and families because of its toll on the health of many women and its influence on pregnancy outcomes and child development. If perinatal depression early in pregnancy is associated with lower amounts of DHA in breast milk, early screening for major depression and the study of ways to improve the DHA status of women might be important steps to reduce the burden of perinatal depression.

\section{Acknowledgments}

We thank the participants in the Pregnancy, Infection, and Nutrition studies, Kathryn Carrier for technical assistance, Kerry-Ann Da Costa for laboratory support, and Diane Kaczor for programming support.

Funding

The authors disclosed receipt of the following financial support for the research and/or authorship of this article: This work was supported by grants from the Eunice Kennedy Shriver National Institute of Child Health and Human Development (HD37584, HD39373), the National Institute of Diabetes and Digestive and Kidney Diseases (DK61981, DK56350), and the National Institute of Environmental Health Sciences (P30ES10126) of the National Institutes of Health, the Carolina Population Center, and as part of the salary-supported activities of extramural staff of the National Institute of Child Health and Human Development.

\section{References}

1. Gaynes, B.; Gavin, N.; Meltzer-Brody, S., et al. Perinatal Depression: Prevalence, Screening Accuracy, and Screening Outcomes. Rockville, MD: Agency for Healthcare Research and Quality; 2005. Evidence report/technology assessment 119. AHRQ publication 05-E006-2

2. Liperoti R, Landi F, Fusco O, Bernabei R, Onder G. Omega-3 polyunsaturated fatty acids and depression: a review of the evidence. Curr Pharm Des. 2009; 15:4165-4172. [PubMed: 20041818]

3. Edwards R, Peet M, Shay J, Horrobin D. Omega-3 polyunsaturated fatty acid levels in the diet and in red blood cell membranes of depressed patients. J Affect Disord. 1998; 48:149-155. [PubMed: 9543204]

4. McNamara RK, Hahn CG, Jandacek R, et al. Selective deficits in the omega-3 fatty acid docosahexaenoic acid in the postmortem orbitofrontal cortex of patients with major depressive disorder. Biol Psychiatry. 2007; 62:17-24. [PubMed: 17188654]

5. Peet M, Murphy B, Shay J, Horrobin D. Depletion of omega-3 fatty acid levels in red blood cell membranes of depressive patients. Biol Psychiatry. 1998; 43:315-319. [PubMed: 9513745]

6. Innis SM. Omega-3 fatty acids and neural development to 2 years of age: do we know enough for dietary recommendations? J Pediatr Gastroenterol Nutr. 2009; 48(suppl 1):S16-S24. [PubMed: 19214053]

7. Lauritzen L, Hansen HS, Jorgensen MH, Michaelsen KF. The essentiality of long chain n-3 fatty acids in relation to development and function of the brain and retina. Prog Lipid Res. 2001; 40:194. [PubMed: 11137568] 
8. Freeman MP, Hibbeln JR, Wisner KL, Watchman M, Gelenberg AJ. An open trial of Omega-3 fatty acids for depression in pregnancy. Acta Neuropsychiatr. 2006; 18:21-24.

9. Freeman MP, Hibbeln JR, Wisner KL, Brumbach BH, Watchman M, Gelenberg AJ. Randomized dose-ranging pilot trial of omega-3 fatty acids for postpartum depression. Acta Psychiatr Scand. 2006; 113:31-35. [PubMed: 16390366]

10. Freeman MP, Davis M, Sinha P, Wisner KL, Hibbeln JR, Gelenberg AJ. Omega-3 fatty acids and supportive psychotherapy for perinatal depression: a randomized placebo-controlled study. $\mathrm{J}$ Affect Disord. 2008; 110:142-148. [PubMed: 18206247]

11. Llorente AM, Jensen CL, Voigt RG, Fraley JK, Berretta MC, Heird WC. Effect of maternal docosahexaenoic acid supplementation on postpartum depression and information processing. Am J Obstet Gynecol. 2003; 188:1348-1353. [PubMed: 12748510]

12. Marangell LB, Martinez JM, Zboyan HA, Chong H, Puryear LJ. Omega-3 fatty acids for the prevention of postpartum depression: negative data from a preliminary, open-label pilot study. Depress Anxiety. 2004; 19:20-23. [PubMed: 14978781]

13. Browne JC, Scott KM, Silvers KM. Fish consumption in pregnancy and omega-3 status after birth are not associated with postnatal depression. J Affect Disord. 2006; 90:131-139. [PubMed: 16325262]

14. De Vriese SR, Christophe AB, Maes M. Lowered serum n-3 polyunsaturated fatty acid (PUFA) levels predict the occurrence of postpartum depression: further evidence that lowered n-PUFAs are related to major depression. Life Sci. 2003; 73:3181-3187. [PubMed: 14561523]

15. Otto SJ, de Groot RH, Hornstra G. Increased risk of postpartum depressive symptoms is associated with slower normalization after pregnancy of the functional docosahexaenoic acid status. Prostaglandins Leukot Essent Fatty Acids. 2003; 69:237-243. [PubMed: 12907133]

16. Rees AM, Austin MP, Owen C, Parker G. Omega-3 deficiency associated with perinatal depression: case control study. Psychiatry Res. 2009; 166:254-259. [PubMed: 19268372]

17. Beck CT. A meta-analysis of predictors of postpartum depression. Nurs Res. 1996; 45:297-303. [PubMed: 8831657]

18. Innis S. Human milk: maternal dietary lipids and infant development. Proc Nutr Soc. 2007; 66:397-404. [PubMed: 17637092]

19. Makrides M, Neumann MA, Gibson RA. Effect of maternal docosahexaenoic acid (DHA) supplementation on breast milk composition. Eur J Clin Nutr. 1996; 50:352-357. [PubMed: 8793415]

20. Hachey DL, Thomas MR, Emken EA, et al. Human lactation: maternal transfer of dietary triglycerides labeled with stable isotopes. J Lipid Res. 1987; 28:1185-1192. [PubMed: 3681142]

21. van Goor SA, Smit EN, Schaafsma A, Dijck-Brouwer DA, Muskiet FA. Milk of women with lifetime consumption of the recommended daily intake of fish fatty acids should constitute the basis for the DHA contents of infant formula. J Perinat Med. 2008; 36:548-549. [PubMed: 18673085]

22. Jensen CL, Maude M, Anderson RE, Heird WC. Effect of docosahexaenoic acid supplementation of lactating women on the fatty acid composition of breast milk lipids and maternal and infant plasma phospholipids. Am J Clin Nutr. 2000; 71 suppl(1):292S-299S. [PubMed: 10617985]

23. Siega-Riz AM, Herring AH, Carrier K, Evenson KR, Dole N, Deierlein A. Sociodemographic, perinatal, behavioral, and psychosocial predictors of weight retention at 3 and 12 months postpartum. Obesity (Silver Spring). 2010; 18:1996-2003. [PubMed: 20035283]

24. Mumford SL, Siega-Riz AM, Herring A, Evenson KR. Dietary restraint and gestational weight gain. J Am Diet Assoc. 2008; 108:1646-1653. [PubMed: 18926129]

25. Radloff LS. The CES-D Scale: a self-report depression scale for research in the general population. Appl Psychol Meas. 1977; 1:385-401.

26. Bligh EG, Dyer WJ. A rapid method of total lipid extraction and purification. Can J Biochem Physiol. 1959; 37:911-917. [PubMed: 13671378]

27. Siega-Riz AM, Bodnar LM, Savitz DA. What are pregnant women eating? Nutrient and food group differences by race. Am J Obstet Gynecol. 2002; 186:480-486. [PubMed: 11904611]

28. Food and Nutrition Board. Dietary Reference Intakes for Energy, Carbohydrate, Fiber, Fat, Fatty Acids, Cholesterol, Protein, and Amino Acids (Macronutrients). A Report of the Panel on 
Macronutrients, Subcommittees on Upper Reference Levels of Nutrients and Interpretation and Uses of Dietary Reference Intakes, and the Standing Committee on the Scientific Evaluation of Dietary Reference Intakes. Washington, DC: Institute of Medicine; 2005.

29. Halbreich U. Postpartum disorders: multiple interacting underlying mechanisms and risk factors. J Affect Disord. 2005; 88:1-7. [PubMed: 15996747]

30. Hibbeln JR. Seafood consumption, the DHA content of mothers' milk and prevalence rates of postpartum depression: a crossnational, ecological analysis. J Affect Disord. 2002; 69:15-29. [PubMed: 12103448]

31. Evans J, Heron J, Francomb H, Oke S, Golding J. Cohort study of depressed mood during pregnancy and after childbirth. BMJ. 2001; 323:257-260. [PubMed: 11485953]

32. Matthey S, Barnett B, Ungerer J, Waters B. Paternal and maternal depressed mood during the transition to parenthood. J Affect Disord. 2000; 60:75-85. [PubMed: 10967366]

33. Birch EE, Hoffman DR, Uauy R, Birch DG, Prestidge C. Visual acuity and the essentiality of docosahexaenoic acid and arachidonic acid in the diet of term infants. Pediatr Res. 1998; 44:201209. [PubMed: 9702915] 

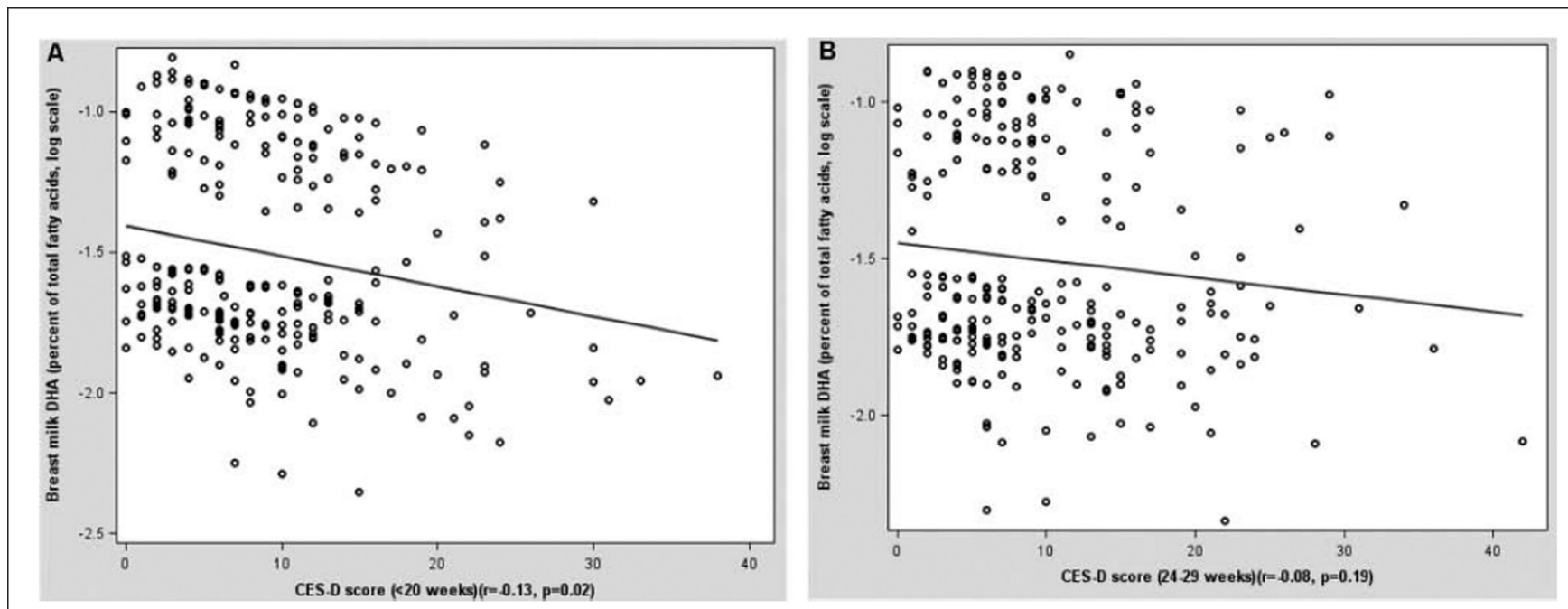

Figure 1.

Breast milk concentration of docosahexaenoic acid (DHA; percentage of total fatty acids, log-transformed) by depressive symptoms score (Center for Epidemiologic Studies Depression Scale [CES-D]), adjusted for age, education, income, smoking, parity, and laboratory: A, < 20 weeks of pregnancy $(n=287) ; B, 24-29$ weeks of pregnancy $(n=287)$. 


\section{Table 1}

Characteristics of Eligible Women in the Pregnancy, Infection, and Nutrition (PIN) and PIN Postpartum Studies who Provided Breast Milk Samples, 2002-2006

\begin{tabular}{|c|c|}
\hline & No. $(\%)$ \\
\hline Overall & $287(100)$ \\
\hline \multicolumn{2}{|l|}{ Maternal education } \\
\hline $0-12$ years & $16(5.6)$ \\
\hline $13-16$ & $135(47.0)$ \\
\hline$>16$ & $136(47.4)$ \\
\hline \multicolumn{2}{|l|}{ Smoking } \\
\hline Yes & $9(3.2)$ \\
\hline No & $269(96.8)$ \\
\hline \multicolumn{2}{|l|}{ Maternal age, y } \\
\hline $17-20$ & $6(2.1)$ \\
\hline $21-30$ & $132(46.0)$ \\
\hline $31-47$ & $149(51.9)$ \\
\hline \multicolumn{2}{|l|}{ Parity } \\
\hline 0 & $150(52.3)$ \\
\hline 1 & $96(33.5)$ \\
\hline $2+$ & $41(14.3)$ \\
\hline \multicolumn{2}{|c|}{ Income (percentage of federal level) } \\
\hline$<185 \%$ & $19(6.7)$ \\
\hline $185 \%-350 \%$ & 47 (16.7) \\
\hline$>350 \%$ & $216(76.6)$ \\
\hline \multicolumn{2}{|l|}{ Laboratory } \\
\hline Minnesota & $171(59.6)$ \\
\hline \multirow[t]{2}{*}{ North Carolina } & $116(40.4)$ \\
\hline & Median (Interquartile Range) \\
\hline \multicolumn{2}{|c|}{ Breast milk fatty acid concentration, $\%$} \\
\hline Total $\omega-3$ & $1.71(0.78)$ \\
\hline Total $\omega-6$ & $17.27(5.13)$ \\
\hline$\omega-6: \omega-3$ & $10.25(3.70)$ \\
\hline Eicosapentaenoic acid & $0.08(0.06)$ \\
\hline Docosahexaenoic acid & $0.20(0.22)$ \\
\hline
\end{tabular}




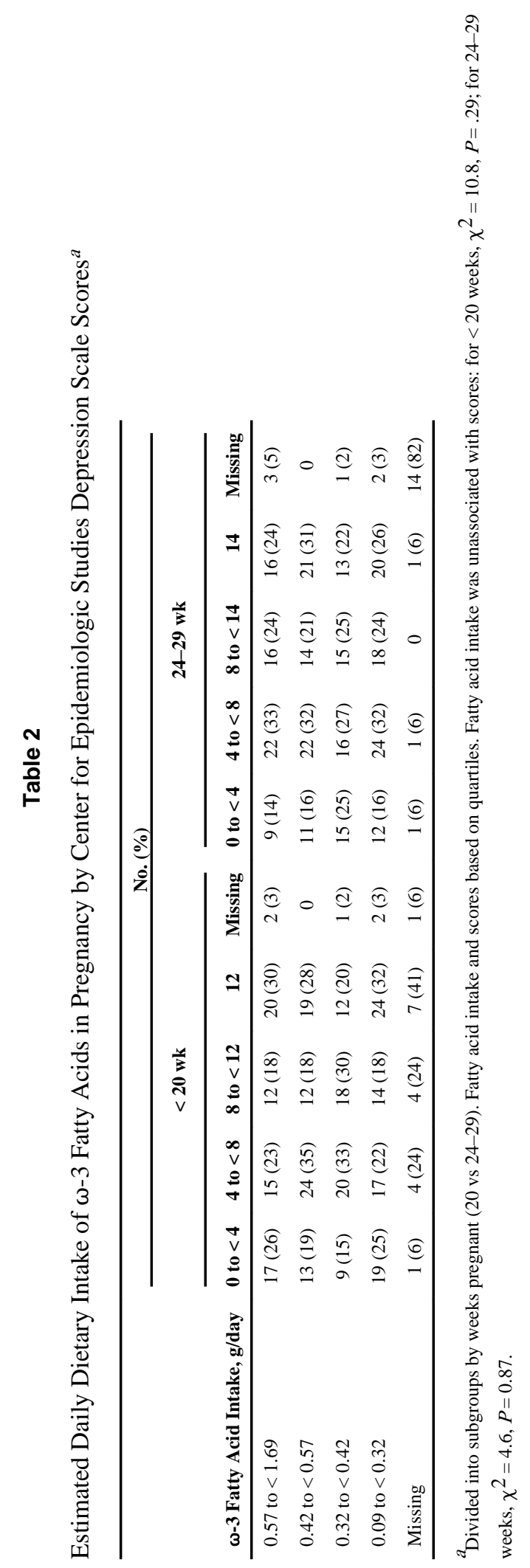




\section{Table 3}

Percentage Change in Geometric Mean Breast Milk Fatty Acid Concentration for a 1-Point Increase in Center for Epidemiologic Studies Depression Scale Score

\begin{tabular}{|c|c|c|}
\hline Fatty Acid $^{a}$ & Unadjusted Model (95\% Confidence Interval) ${ }^{b}$ & Adjusted Model (95\% Confidence Interval) ${ }^{c}$ \\
\hline \multicolumn{3}{|l|}{$<20 \mathrm{wk}$, pregnancy } \\
\hline Total $\omega-3$ & $-0.67(-1.27,-0.07)$ & $-0.55(-1.21,0.13)$ \\
\hline Total $\omega-6$ & $-0.21(-0.57,0.16)$ & $-0.30(-0.691 .01)$ \\
\hline$\omega-6: \omega-3$ & $0.04(-0.03,0.10)$ & $0.01(-0.06 .0 .9)$ \\
\hline Eicosapentaenoic acid & $-0.86(-1.73,0.04)$ & $-0.31(-1.28,0.67)$ \\
\hline Docosahexaenoic acid & $-1.32(-2.19,-0.46)$ & $-1.15(-2.12,-0.19)$ \\
\hline \multicolumn{3}{|l|}{ 24-29 wk, pregnancy } \\
\hline Total $\omega-3$ & $-0.32(-0.90,0.27)$ & $-0.14(-0.76,0.48)$ \\
\hline Total $\omega-6$ & $-0.07(-0.41,0.26)$ & $-0.19(-0.55,0.16)$ \\
\hline$\omega-6: \omega-3^{d}$ & $0.2(-0.04,0.08)$ & $-0.01(-0.08,0.05)$ \\
\hline Eicosapentaenoic acid & $-0.73(-1.57,0.12)$ & $-0.18(-1.05,0.70)$ \\
\hline Docosahexaenoic acid & $-0.69(-1.54,0.16)$ & $-0.27(-1.16,0.61)$ \\
\hline \multicolumn{3}{|c|}{ Regression models included log-transformed fatty acid variables. The results presented were backtransformed. } \\
\hline \multicolumn{3}{|c|}{$b_{\text {Unadjusted models included a variable for the laboratory analyzed the sample. }}$} \\
\hline \multicolumn{3}{|c|}{$\begin{array}{l}c \text { Adjusted models included education, smoking, maternal age, parity, income, estimated daily dietary intake of } \omega-3 \text { or } \omega-6 \text { fatty acids (depending } \\
\text { on the model), and laboratory. }\end{array}$} \\
\hline
\end{tabular}

